\title{
CTA - A Project for a New Generation of Cherenkov Telescopes
}

\author{
Michele Doro* \\ University of Padova E INFN Padova, v. Marzolo 8, 35131 Padova, Italy
}

\begin{abstract}
Gamma-rays provide a powerful insight into the non-thermal universe and perhaps a unique probe for new physics beyond the standard model. Current experiments are already giving results in the physics of acceleration of cosmic rays in supernova remnants, pulsar and active galactic nuclei with a hundred sources detected at very-high-energies so far. Despite its relatively recent appearance, very high-energy gamma-ray astronomy has proven to have reached a mature technology with fast assembling, relaD tively cheap and reliable telescopes. The goal of future installation is to increase the sensitivity by a factor ten compared to current installations, and enlarge the energy domain from few tens of $\mathrm{GeV}$ to a hundred TeV. Gamma-ray spectra of astrophysical origin 'are rather soft thus hardly one single size telescope can cover more than 1.5 decades in energy, therefore an array of telescopes of $2-3$ different sizes is required. Hereafter, we present design considerations for a Cherenkov Telescope Array (CTA), a project for a new generation of highly automated telescopes for gamma-ray astronomy. The status of the project, technical solutions and an insight in the involved physics will be presented.
\end{abstract}

\section{Introduction}

\subsection{Gamma-ray astronomy as a probe for cosmic rays.}

Despite its origin goes back to only few decades ago, groundbased gamma-ray astronomy has already demonstrated to be a mature scientific technique to probe non-thermal phenomena in the universe, where cosmic ray $(\mathrm{CR})$ particles are accelerated to extremely high energies. CRs of non-thermal origin can be accelerated either directly at the place of origin, for example nearby the surface of a fast rotating pulsar, or may gain energy in cosmological times through interaction with irregular cosmic magnetic fields or shock wave fronts. CRs cover a vast range of energies, from $10^{9}$ to $10^{21} \mathrm{eV}$. Below $100 \mathrm{GeV}$ the CR interaction with the solar wind is efficient and they are absorbed. Their flux covers more than 32 orders of magnitude, and they are distributed roughly according to a power-law of spectral index -3 . There are many publications about CRs, but still a conclusive evidence about how and where they are accelerated, is missing [1]. CRs are mainly composed of protons and helium, with smaller percentages of heavier elements and electrons. Due to the rapidly falling fluxes of CRs, it is experimentally difficult to cover an energy band larger than few decades in energy by a single experiment. Therefore different experimental techniques are needed for different energy ranges. They are typically investigated with balloon-borne calorimeters and ground-based detectors. For a recent review, see, e.g., Ref. [2].

After the second half of last century, it was recognized that gamma-rays (GRs) could provide a useful tool to investigate the origin of CRs. First of all, GRs are neutral, thus they travel undeflected by inter-stellar or inter-galactic magnetic fields and

\footnotetext{
* for the CTA consortium.

Email address: michele.doro@pd.infn.it (Michele Doro)
}

therefore trace back their origin, and second, they interact with local radiation/dust fields thus providing useful information on the morphology of the emission region.

\subsection{Scientific targets}

As in the case of CRs, it is not possible to construct a single observatory which could cover the entire GR spectrum because of the rapidly falling flux with GR energies. From an experimental point of view, two sub-ranges are defined at high energy (HE; MeV-GeV) and very-high energy (VHE; GeV-TeV). For many sources the combined power emitted at these energies overcome the total power emitted at other wavelengths, and therefore HE and VHE astrophysics has attained large attention over the last years [2].

The goals of GR astronomy could be divided in three categories: the galactic targets, like pulsar and pulsar-wind nebulae (PWN), supernova remnants (SNR) and star-active region like OB-associations or binary systems. Another fundamental target in this family is the galaxy center (GC). Among the extragalactic targets, stand the active galactic nuclei (AGN), particularly blazars and radio-galaxies. Galaxy clusters, starburst and merging galaxies are also interesting targets. Finally, gamma-ray bursts (GRBs) are also among this category. There is a third family of observation about fundamental physics, which can be studied within GR astronomy. At first place is the study of dark matter (DM) annihilation or decays, which could have very clear GR signatures. The extragalactic background light (EBL) can be studies through the GR absorption from distant targets to understand the universe transparency. Other questions like axion physics, Lorentz invariance violation, antiparticle asymmetry can also be addressed. For a recent review, see, e.g., Ref. [3]. 


\subsection{The IACT technique}

Very high energy GRs impinging on the earth, interact with atmospheric nuclei and generate an electromagnetic shower. The showers extend over several kilometers in length and few tens to hundreds of meters in width, and their maximum is located at $8-12 \mathrm{~km}$ altitude, in case of vertical incidence. For gammas below around $100 \mathrm{TeV}$, the shower particles stop high up in the atmosphere, and can not be directly detected at ground. However, a sizeable fraction of the charged secondary shower particles, mostly electrons and positrons in the shower core, move with ultra-relativistic speed and emit Cherenkov light. This radiation is mainly concentrated in the near UV and optical band and therefore passes mostly unattenuated to the ground, with minor losses due to Rayleigh and Mie scattering and Ozone absorption. Imaging Atmospheric Cherenkov Telescopes (IACTs) reflect the Cherenkov light at the focal plane where a multipixel camera records the shower image. The technique was pioneered by the Whipple experiment, which first detected the Crab Nebula at VHE, in 1989.

\section{Current IACTs. Technologies and Selected Results}

\subsection{Current experiments}

Currently, the world largest ground-based IACTs are HESS, MAGIC and VERITAS1 (Figure 1). HESS is an array of 4 clone telescopes, each of $12 \mathrm{~m}$ diameter, located in the Gamsberg mountain in Namibia and is operating since 2003 with a very high scientific impact. A fifth telescope, dubbed HESSII, of $28 \mathrm{~m}$ diameter, is under construction at the center of the array, and its completion is foreseen for 2010. MAGIC has operated since 2004 with a single dish of $17 \mathrm{~m}$ diameter and parabolic profile in the Canary island La Palma in Spain. Despite the use of a single reflector does not guarantee the sensitivity of an array, its world-largest dish allowed to reach the lowest energy threshold of the IACT technique, performing for the first time observation below $100 \mathrm{GeV}$ with this technique. Recently MAGIC has detected the first ever observed GR pulses at $25 \mathrm{GeV}$ from the Crab pulsar [4]. Recently this year, a second clone MAGIC telescope, dubbed MAGIC II, has been inaugurated. The use of the stereoscopic system will allow MAGIC phase-II to reach the sensitivity of larger arrays. A more recent experiment was started in the Arizona desert in USA, following the successful experience of the Whipple experiment. VERITAS has soon reached the expected performance, with a sensitivity comparable to HESS and is starting to collect important scientific results.

\subsection{IACT, a well proven technology.}

Despite its origin traces back to the late 70's, one can allege that IACT has already reached a mature technological development. In the following, the basic features will be discussed. Mounting. HESS, MAGIC and VERITAS have an alt-azimuth mount, but while the former two have structures rotating on

\footnotetext{
${ }^{1}$ www.mpi-hd.mpg.de/HESS, $\quad$ wwwmagic.mppmu.mpg.de, $\quad$ veritas.sao.arizona.edu
}

wheels, VERITAS has a sole central mast and therefore an easier design. HESS is built from stainless steel while MAGIC is made of lighter carbon-fiber reinforced-plastic (CFRP) tubes. Mirrors. The demands for the quality of mirror facets for IACTs are quite less challenging than for optical telescopes, because IACTs focus on the intrinsic-aberrated Cherenkov light from atmospheric showers. On the other hand, the facets are exposed to the environment and must be robust. A classical solution is the use of quartz-coated aluminized glass substrate. An innovative solution was used by MAGIC, based on all-aluminum diamondmilled sandwiches with hexcell honeycomb interspaced. This technique has proven to produce mirrors with reflectivity loss more than 5 times smaller compared to standard techniques. MAGIC II is also featuring novel glass-aluminum sandwich mirror facets, whose performance is under investigation and with interesting prospects for the future. Focal-plane instrumentation. HESS, MAGIC and VERITAS have a focal-plane instrumentation composed of a multi-pixel camera of photomultiplier tube (PMTs; respectively 960, 576, 1038, 499 for HESS, MAGIC-I, MAGIC-II and VERITAS). PMTs are an optimal solution due to their high gain and fast read-out. A drawback is their limited photon conversion efficiency for Cherenkov photons, currently about 20-25\%. More performing devices are under research, as will be discussed later. Read-out electronics. The short duration of Cherenkov signals demands at least a $\mathrm{MHz}$ sampling capacity and a signal forming, whereas $\mathrm{GHz}$ sampling is already in use in MAGIC and HESS. Such technology is continuously evolving and research is ongoing. Trigger system. The trigger system is multifold: the basic discrimination is at the photoelectrons level to adjust to different illumination conditions (moon, galactic or extragalactic targets); a second-level trigger is typically topological when a cluster of PMTs are activated. This allows to reject spurious events from the light of the night sky. Other triggers are used to synchronize telescopes in the array or make more advance logical trigger. Calibration. Despite an exact energy calibration is impossible for IACT because of a missing calibrated GR source in the sky 2 and because of the varying conditions of the atmosphere, several instrumental calibration methods are normally applied. In the future, particular interest will be focussed on improving devices and methods for calibration to reduce systematic biases in particular regarding the energy resolution.

\subsection{Selection of scientific results}

Interesting reviews of scientific results of GR astrophysics can be found in Refs. [2, 3].

\section{Towards a precision gamma-ray astronomy. Physics motivation for CTA.}

Despite the promising achievements from the current generation of IACTs, there is a number of limitations that the future generation will overcome: a) IACTs of current generation are

\footnotetext{
${ }^{2}$ This fact will be strongly mitigated once IACT data could be crosscalibrated with satellite GR detector data (e.q Fermi).
} 


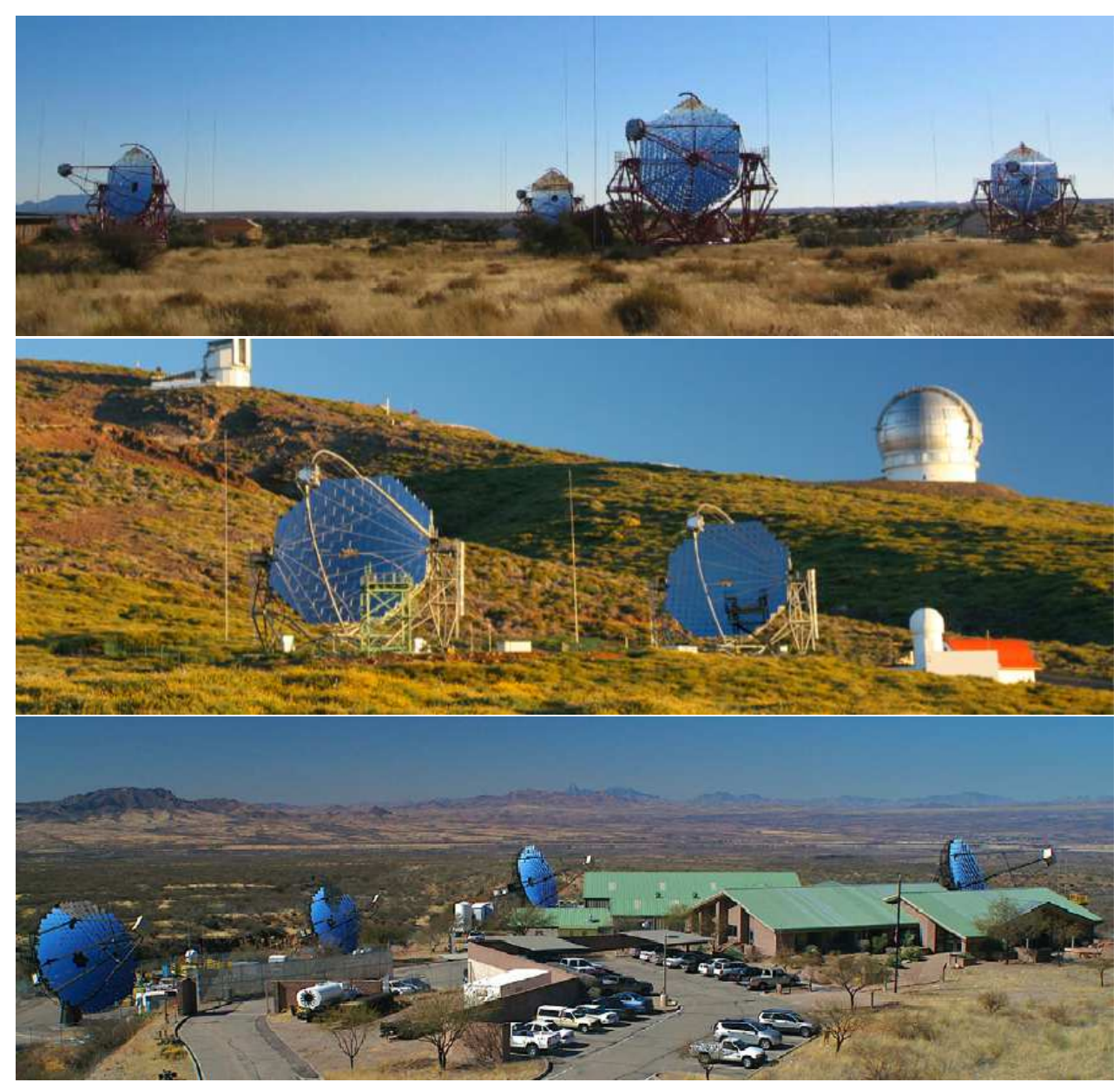

Figure 1: From top to bottom, the HESS array of four telescopes located in Namibia; the MAGIC array of two $17 \mathrm{~m}$ diameter telescopes located in the Canary island La Palma; the recently completed four IACT array VERITAS at Mt Hopkins, Arizona.

sensitive in a limited energy range, from $100 \mathrm{GeV}$ to $50 \mathrm{TeV}$. At the lower end, IACTs are limited by the background from atmospheric hadronic showers. At the high end, the limit is posed by insufficient statistics; $b$ ) Due to the lack of a calibrated cosmic GR source, IACT spectral reconstruction is limited by systematic bias and statistical uncertainties on the energy reconstruction; $c$ ) They have a limited aperture, with typical field of view (FOV) of the order of $3-5^{\circ}$ diameter; $d$ ) They have a limited angular resolution which currently states around few arcmin; $e$ ) They have limited collection area; $f$ ) They are rather poorly automatized.

On the other hand, from a physical point of view, there are strong arguments to improve in the following aspects:

1. Decrease the energy threshold to few tens of $\mathrm{GeV}$

2. Acquire sensitivity beyond $50 \mathrm{TeV}$

3. Increase sensitivity in the bulk range $100 \mathrm{GeV}, 50 \mathrm{TeV}$

4. Improve energy and angular resolution

The accomplishment of the above goals is intimately related to the overcome of the technological questions discussed at the beginning of this section. For this reason, a new generation of IACT is under design now with expected performance well above the current generation, as shown in Figure 2. We believe that with CTA we are heading toward a new era of precision $G R$ astronomy. In the following, the above arguments are discussed in more detail.

\subsection{Low-energy physics (sub-50 GeV)}

There is a lot of expectations in the GR physics below $50 \mathrm{GeV}$. MAGIC has opened the field of sub-100 GeV GR astronomy, although this is currently done only with poor significance. Observations with larger sensitivity in this region will have several important consequences for galactic and extragalactic physics, and maybe for fundamental physics. The expectations in term of sensitivity are shown in Figure2.

Pulsars are compact objects, residual of exploded massive stars, with extreme physical conditions in their proximity: high magnetic field $\left(10^{12}-10^{15}\right.$ Gauss) and strong particle and radiation winds. They are observable at all wavelengths, particularly in the radio band, since many years, with a catalog of thousand objects. On the other hand, only around 60 pulsars were recently observed by Fermi at HE GRs [5], and only the Crab pulsar was observed by MAGIC at VHE energies [4]. This is related to the fact that a cutoff in the emission is expected in the region between few $\mathrm{GeV}$ and few tens of $\mathrm{GeV}$. By studying this 


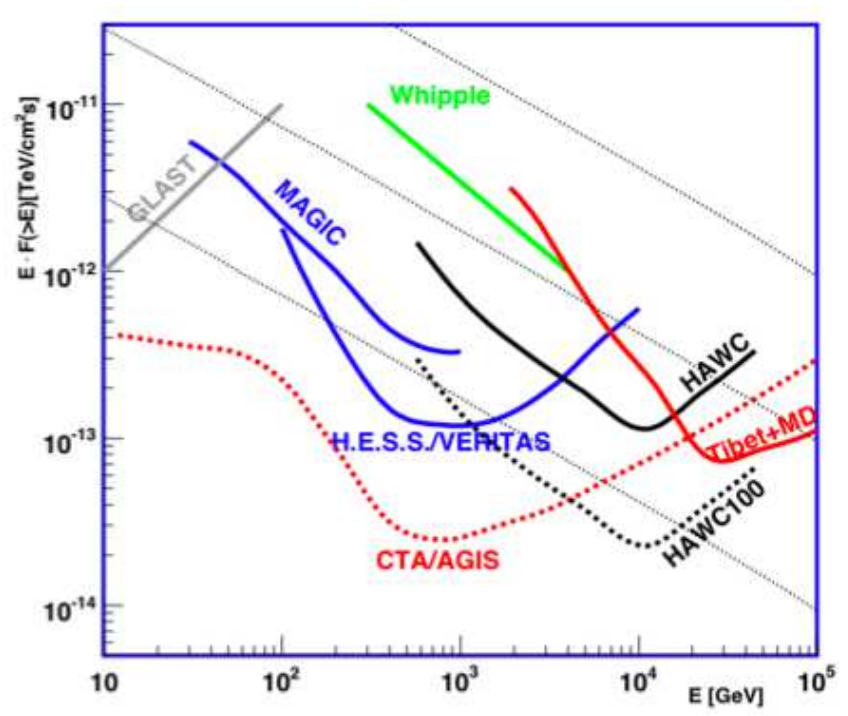

Figure 2: Integral sensitivity for a Crab-like spectrum for several current IACT and expected for CTA/AGIS $(5 \sigma, 50 \mathrm{~h})$ and Fermi/GLAST $(5 \sigma, 1 \mathrm{yr})$.

energy band, CTA will provide the final answer to the acceleration mechanism in pulsar, because the exact location of the cutoff strongly depend on the pulsar acceleration model (e.g., higher energy cutoffs foresee acceleration far from the pulsar surface) and the high energy tail of the Fermi spectra will be completed and cross-checked with higher significance.

Farther from the pulsar core, the pulsar particle wind is typically able to sweep up the surrounding medium through shock mechanism and forming the PWN. Ultrarelativistic electrons inside the nebula emits synchrotron GRs in the HE and VHE regime as a result of the interaction with local magnetic fields. The exact location of the synchrotron peak is still unknown and may depends on the exact feature of the emitter. Low-energy studies will permit to fully characterize this important radiation loss mechanisms in the nebula and the interaction with the surrounding medium.

Even farther away from the remaining of a collapsing supernova star, one can find the remnants of the material ejected at the explosion. These are called SNRs. They are expanding clouds which propagate at fast velocity. All the charged particles inside the clouds are heated up and accelerated by statistical mechanisms described by the Diffusive Shock Acceleration (DSA) model [6] based on the second Fermi acceleration mechanism. Those models predict acceleration of electrons and/or light hadrons. While the electronic acceleration has several evidences, there is not yet a clear indication that hadronic acceleration takes place at SNRs. A clearer view will come from low-energy studies because the predictions for the two emissions differ substantially at these energies.

The same argument for acceleration mechanism applies in the case of AGNs, particularly in the case of the steep spectrum blazars. Finally, the importance of low-energy studies should not be underestimated, to complete the Fermi catalog of HE emitters in the VHE tail with possibly a larger significance than what Fermi can obtain. This will play a fundamental role in the modelization of the source emission, and will provide a unique way to understand the nature of the tens of yet unidentified Fermi sources [5].

\subsection{High-energy physics (above $50 \mathrm{TeV}$ )}

It is strongly believed that typical GR spectra of astrophysical source have a bimodal distribution with one peak at lower energies to synchrotron emission from charged particles, and a secondary peak at higher energies due to inverse Compton scattering of VHE electrons on seed IR photons. In case of galactic objects, at VHE one may expect to observe power-law GR spectra with cutoffs due to intrinsic mechanisms. On the other hand, GRs from distant blazars suffer a severe attenuation after pair production with local IR-UV photons of the extragalactic background light (EBL). In all current IACT data, the evidences for spectral cutoffs both for extragalactic and galactic objects are rather poor. There is no simple justification for this evidence. It is possible however that cutoffs are placed at higher energies.

CTA will explore this region with unprecedented significance. This will allow to understand the acceleration mechanism in galactic objects like SNRs, and discriminating the hadronic vs leptonic models. Hadrons are expected to produce higher energy GR due to the faster electron energy loss. Additional interesting targets will be understood in this domain. For example, the nature of the ultrarelativistic jets of micro-quasars and of binary objects with massive infall of materials will be clarified.

Finally, detecting eventual flares from distant object like AGNs at super-TeV energies, would allow to increase the prospects for a detection of Lorentz invariance violation.

\subsection{TeV-regime (0.1-50 TeV). Bulk research field.}

The greater effort for CTA will be concentrated in boosting the instrumental sensitivity of at least a factor 10 in the actual sensitive region of current IACTs, i.e. between $100 \mathrm{GeV}$ and $50 \mathrm{TeV}$, reaching a level of $10^{-3} \mathrm{C}$.U 3 sensitivity in this range (see Figure 2). This will promote the GR astrophysics science to the level of GR astronomy. In fact, for the first time, a full VHE sky coverage will be obtained, with a thousand of new VHE GR sources expected. In parallel, the first VHE GR catalog of emitters will be released, making a big step toward a complete understanding of the non-thermal emission scenario. Entire classes of sources could be understood once their acceleration mechanisms will be revealed.

The increase in sensitivity will in fact not only have the consequence of requiring shorter observation time. An increase in sensitivity will allow to: $a$ ) increase the possibility for followup observation and finer resolution time-variability, and $b$ ) improve the resolution for morphological observation. Actual telescopes are at best sensitive enough to detect variations on the time scale of minutes. By increasing the sensitivity of a factor 10, a sub-minute resolution will open the door to understand the complex phenomena of GR flares, directly connected to the acceleration mechanisms and the local environment. Also of utmost importance is the morphological studies of extended GR

${ }^{3} 1$ C.U. $($ Crab Unit $)=1.5 \times 10^{3}(\mathrm{E} / \mathrm{GeV})^{2.58} \mathrm{ph} \mathrm{cm}^{-2} \mathrm{~s}^{-1} \mathrm{TeV}^{-1}$ 
emission from galactic sources like SNRs. In this case, a uniform sensitivity must be guaranteed over the entire FOV of the instruments and therefore an overall gain in sensitivity must be accomplished. Morphological studies allow a preciser comparison with other wavelengths and a clearer view of the local interactions.

\section{General technical ideas on CTA}

To accomplish with the physical requirements specified in the previous section and to maintain an overall high technical performance, the CTA concept is based on few general ideas:

1. Increase the array from 4 to around 100 telescopes;

2. Distribute them over a large area $\left(1-10 \mathrm{~km}^{2}\right)$;

3. Make use of telescopes of $2-3$ different sizes;

4. Take advantage of well-proven technology of current IACTs;

5. High automatization and remote operation;

6. Run array as observatory and open to astronomer community.

\subsection{Basic array design}

The design of the array is artistically depicted in Figure 3. The CTA project is being designed both to provide an expansion of the energy range down to a few tens of $\mathrm{GeV}$ and up to about $100 \mathrm{TeV}$ and with at least 10 times improvement in sensitivity compared to current installations. This can only be achieved by combining $a$ ) many telescopes distributed over a large area of at least $1 \mathrm{~km}^{2}$ and $b$ ) using telescopes of different sizes. CTA is planned to comprise about a hundred telescopes of $2-3$ different sizes: several small size telescopes (SST) of $6 \mathrm{~m}$ diameter, several medium size telescopes (MST) of $12 \mathrm{~m}$ and few large size telescopes (LST) of $23 \mathrm{~m}$ diameter. However, the number of the telescopes, their size, their configuration and the overall performance are still under investigation and the final layout will come out after Monte Carlo optimization.

Few LSTs should catch the sub-100 GeV photons thanks to their large reflective area. To maintain the time stamp of the showers, they will have probably a parabolic shape. To avoid the intrinsic optical aberrations due to this profile, LSTs will probably have limited size FOV $\left(3-4^{\circ}\right)$ while large telescope $f / D>1.2$ ratio presents the technical challenge of displacing the camera at more than $28 \mathrm{~m}$ from the reflector. Technologically, the LST will be the most challenging telescope. A design is current under development.

Several tens of MSTs will perform the bulk TeV search. Those telescopes will come from the well-proven experience of HESS and MAGIC collaborations. The main goal is to reduce the costs and maintenance activities. They will constitute the core of the array, and will perform the fundamental task of vetoing the LST triggers to reduce the hadronic background. Several different designs are currently taken into account and the construction of the first prototypes is expected in few years from now.

Finally, several tens of SSTs will complete the array to perform the super-TeV search. They will be very simple in construction, contributing to a small percentage of costs of the full array, and distributed in between and around the core array of MSTs.

\subsection{Improved angular and energy resolution}

The improvement of energy and angular resolution is also a very important point. The angular resolution should be kept as low as possible in the entire FOV. Current theoretical limits are discussed in [7]. This has the important impact of a) Avoiding the source confusion; b) Contributing in the discrimination between acceleration of hadrons and leptons. They have different free-streaming lengths and the GR emission is strongly shaped by local interactions; c) Possibility to better cope with data at other wavelengths. All this will give key information to discriminate acceleration mechanisms in SNRs, PWNs, binaries, micro-quasar and the GC. An improved angular resolution can be obtained with performing optical system and by keeping optical aberrations low.

An increase in energy resolution (down to $10 \%$ ) has a direct consequence in boosting the capability to observe cutoffs such those expected in pulsars and EBL-absorbed AGNs. Cutoffs are also expected in GR spectra from DM candidates at the DM mass. Finally, a better energy resolution can provide higher quality measurement of Lorentz invariance. This can be achieved by an overall improvement both in the calibration of the detector (light sensor, photon conversion, etc.) and in a deeper monitoring of the atmosphere through transparency instrumentation, like LIDARs.

\subsection{Subsystems}

From the technological point of view, the major effort is currently concentrated on the working packages responsible for telescope design, mirror facets development, electronic and focalplane instrumentation. Different projects for SSTs, MSTs and LSTs are under design at several institutes to stimulate competition and technological development. Mirrors constitute an important challenge because they contribute to a sizeable part of the costs. A major effort is ongoing to build fast assembling, reliable mirrors with reduced optical degradation with time. A review of the different techniques under study can be found in Ref. [8]. The focal-plane instrumentation also represents a challenge in technology and cost. The current baseline design envisages the use of high conversion efficiency PMTs. Studies are ongoing also on GaAsP hybrid photon detectors (HPD) and Geiger avalanche photon detectors (GAPD).

\subsection{Operation modes}

The trigger systems will be flexible for different operation modes. In Figure 4 some observation modes are shown. In the deep field mode, all telescopes will be pointed to the same sky position to maximize the sensitivity. In a more flexible mode, a fraction or two of the telescopes could point to different positions, with few telescopes making follow-up observation of single sources as to monitor blazar activity for example. Finally, telescopes could be operated in wide-FOV mode, pointed at slightly off-centered region, to perform an all-sky scan. 


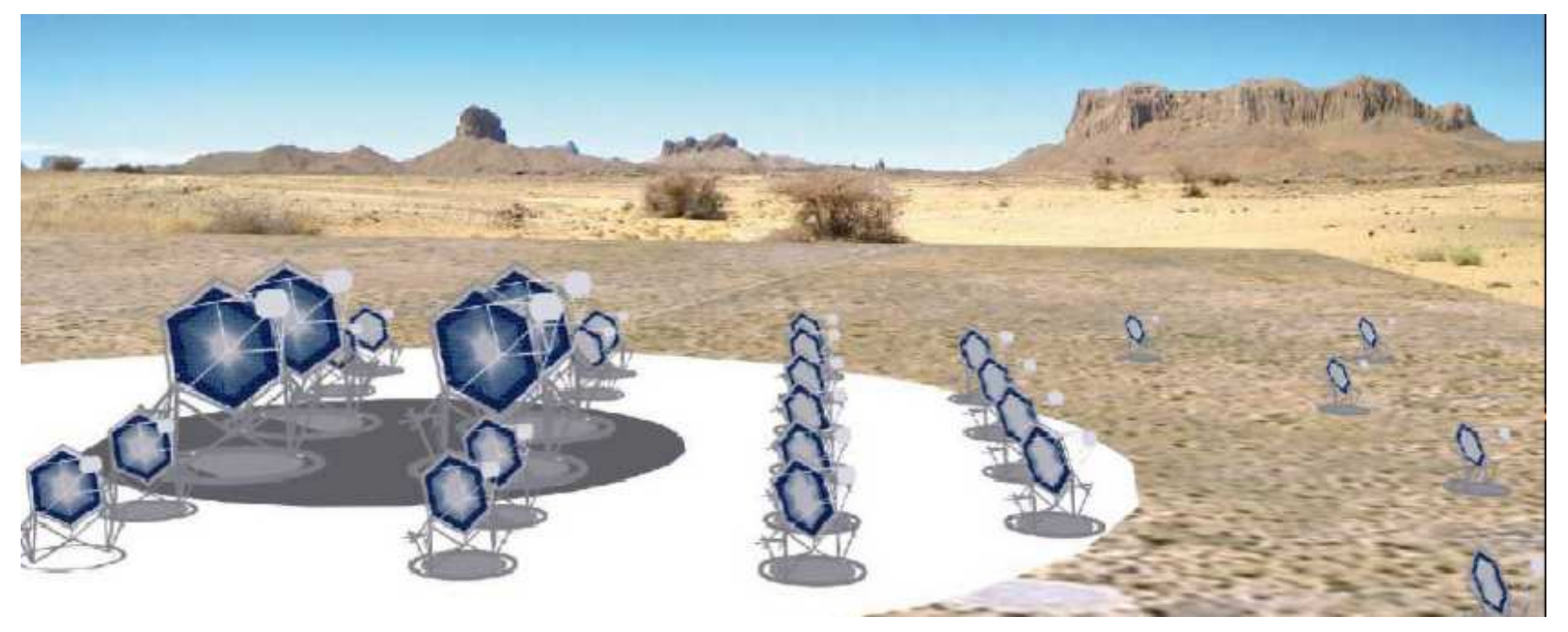

Figure 3: Artistic view of the compound different size telescopes CTA system. The area coverage is of $1-10 \mathrm{~km}^{2}$.
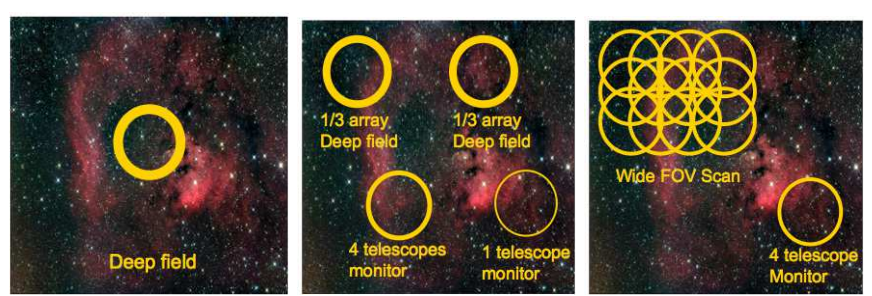

Figure 4: Possible operation modes for CTA. From left to right: deep field, flexible and wide field modes. More details in the text.

\section{The CTA consortium}

The CTA consortium is a partnership between the HESS and MAGIC collaborations plus several European institutes and recent interest from world-wide institutions. Activities are coordinated and discussed with the US AGIS (Advanced Gammaray Imaging System) scientists, who work on a similar project. The consortium hosts already around 50 institutes, 14 countries and 300 scientists. Regular meetings take place since 2007. The project will be run as observatory.

The CTA consortium has organized in several working packages (WPs) each performing research on separated topics. There are 12 WPs: MGT, PHYS, MC, SITE, MIR, TEL, FPI, ELEC, ATAC, OBS, DATA, QA (standing for Management, Physics, Monte Carlo, Site, Mirror, Telescope, Focal-Plane instrumentation, Electronics, Atmospheric Transmission and Calibration, Observatory, Data, and Quality Assets).

A tentative schedule of activities (funds dependent) is reported in Table 1

\section{Summary}

For a new generation of IACTs, it is mandatory to: extend energy range from few tens of $\mathrm{GeV}$ to $100 \mathrm{TeV}$, improve sensitivity and energy resolution, have a larger FOV and better angular resolution, operate as observatory, perform multiwavelength observations. In 5+ years from now, CTA will

\begin{tabular}{l|lllllll}
\hline & '09 & '10 & '11 & '12 & '13 & '14 & '15 \\
\hline Site exploration & & & & & & & \\
Array layout & & & & & & & \\
Telescope design & & & & & & & \\
Component protot. & & & & & & & \\
Array prototype & & & & & & & \\
Array construction & & & & & & \\
Partial operation & & & & & & & \\
\hline
\end{tabular}

Table 1: CTA tentative schedule of activities.

open the era of precision GR astronomy, with many new galactic and extragalactic objects found. CTA may answer longstanding questions on cosmic-rays: where galactic and extragalactic CRs are accelerated, how CRs are accelerated (hadrons/leptons, jets, magnetic irregularities, etc.). In addition, CTA may answer fundamental physics like DM, Lorentz invariance, universe transparency, photon-axion oscillation.

Acknowledgement. I would like to thanks M. Gaug, N. Godinovic, B. Khelifi and M. Persic for useful comments.

\section{References}

[1] Ginzburg, V. L. and Syrovatskii, S. I., The Origin of Cosmic Rays, New York, Macmillan, 1964

[2] Aharonian, F. et al, Rept. Prog. Phys., 71, 2008, 096901

[3] Buckley, J. et al., The Status and future of ground-based TeV gamma-ray astronomy. American Physical Society., 2008.

[4] Aliu, E. et al. [MAGIC coll.], Detection of pulsed gamma-ays above 25 GeV from the Crab pulsar, Science, 322, 1221-1224, 2008.

[5] Abdo, A. A. et al. [Fermi LAT Collaboration], Fermi Large Area Telescope Bright Gamma-ray Source List, Astrophys. J. Suppl. 183 (2009) 46 arXiv:0902.1340 [astro-ph.HE]].

[6] Drury, L., An introduction to the theory of diffusive shock acceleration of energetic particles in tenuous plasmas., Rept. Prog. Phys., 46:973, 1983.

[7] W. Hofmann, arXiv:astro-ph/0603076

[8] Doro, M., Mirror Facet Technologies for the Telescopes of the CTA Observatory, Proceedings of the $31^{\text {st }}$ ICRC, Łódź 2009 